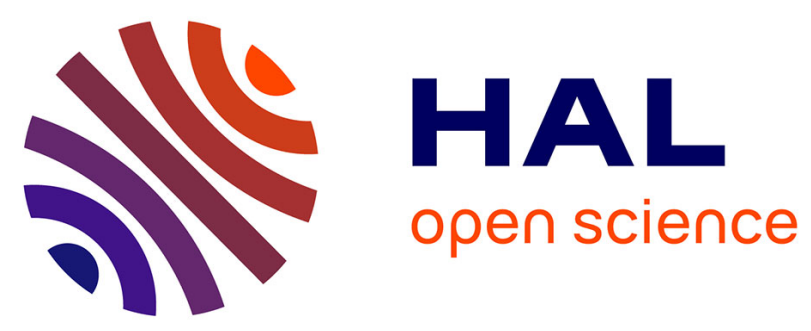

\title{
Calorimetric analysis of coarse-grained polycrystalline aluminum by IRT and DIC
}

\author{
Li Li, Félix Latourte, Jean Michel Muracciole, Laurent Waltz, Laurent
}

Sabatier, Bertrand Wattrisse

\section{- To cite this version:}

Li Li, Félix Latourte, Jean Michel Muracciole, Laurent Waltz, Laurent Sabatier, et al.. Calorimetric analysis of coarse-grained polycrystalline aluminum by IRT and DIC. Quantitative InfraRed Thermography Journal, 2015, 10.1080/17686733.2015.1022354 . hal-01177063

\section{HAL Id: hal-01177063 \\ https://hal.science/hal-01177063}

Submitted on 16 Jul 2015

HAL is a multi-disciplinary open access archive for the deposit and dissemination of scientific research documents, whether they are published or not. The documents may come from teaching and research institutions in France or abroad, or from public or private research centers.
L'archive ouverte pluridisciplinaire HAL, est destinée au dépôt et à la diffusion de documents scientifiques de niveau recherche, publiés ou non, émanant des établissements d'enseignement et de recherche français ou étrangers, des laboratoires publics ou privés. 
To appear in Quantitative InfraRed Thermography

Vol. 00, No. 00, , 1-17

\title{
Calorimetric analysis of coarse-grained polycrystalline aluminum by IRT and DIC
}

Li LI ${ }^{a, *}$, Félix Latourte ${ }^{b}$, Jean-Michel Muracciole ${ }^{a, c}$, Laurent Waltz $^{a, c}$, Laurent Sabatier ${ }^{a, c}$ and Bertrand Wattrisse ${ }^{a, c}$

${ }^{a}$ Laboratoire de Mécanique et Génie Civil (LMGC), Montpellier 2 University, CNRS, France;

${ }^{b}$ EDF R\&D, MMC Dept., Moret-sur-Loing, France; ${ }^{c}$ Laboratoire de Micromécanique et d'Intégrité des Structures (MIST), IRSN-CNRS-Montpellier 2 University, France

(Received: 24 June 2014; Accepted: 13 February 2015)

\begin{abstract}
A novel method is presented in this paper which aims at achieving grain scale energy balances at finite strain in mechanically-loaded polycrystalline metallic specimens. For this purpose, two complementary imaging techniques were used to investigate material thermomechanical behaviour: Digital Image Correlation (DIC) and InfraRed Thermography (IRT) to separately reach the kinematic and the thermal responses of the material. A calorimetric analysis can be conducted by combining these techniques. The aim of this paper is to present and to validate a novel IR data processing method that can be used to perform local thermal field measurements. The procedure was validated on numerical data associated with the response of aluminum polycrystalline aggregates.
\end{abstract}

Keywords: InfraRed Thermography (IRT); Polycrystalline materials; Synthetic image generation; Numerical validation.

\section{Introduction}

Polycrystalline materials are solids composed of an aggregate of crystalline grains of varying size and orientation. It is well known that, during macroscopic monotonic tensile loading, the grain to grain disorientation and the intrinsic anisotropy of crystal plasticity leads to strong heterogeneities in the material plastic response [1, 2], and consequently to a heterogeneous thermal distribution due to thermomechanical coupling effects or to plastically induced self-heating [3, 4].

Heterogeneous phenomena on mechanical and thermal fields have been widely studied since the late 1970s using imaging techniques such as InfraRed Thermography (IRT) $[5,6]$, while Digital Image Correlation (DIC) has been investigated over the last two decades $[7,8]$. With the progress achieved in the field of optical techniques associated with quantitative image processing, the quality, resolution and accuracy of displacement and temperature full-field measurements have improved tremendously. The development of automated Electron BackScattered Diffraction (EBSD) imaging techniques has also contributed to better monitoring of microstructure variations (such as local lattice rotation, grain morphology changes, vanishing of low angle boundaries) during plastic strain [9-11].

\footnotetext{
*Corresponding author. Email: li.li@univ-montp2.fr
} 
Grain scale experimental energy balances are required to assess local transformation energy variations, so as to gain greater insight into the local thermomechanical signature of material deformation mechanisms, in order to be able to propose or validate thermomechanically consistent crystal plasticity models. Few published studies have been devoted to the experimental determination of energy balances at the grain scale in metallic materials [12-17].

A novel IR data processing method is proposed in this paper to perform thermal and calorimetric measurements needed to achieve a local energy balance within each grain. The key feature of this method is that intergranular and intragranular effects can be explicitly considered in the data processing procedure. In this paper, before directly applying the aforementioned data processing method to an experimental case, an in-depth validation involving micro and macro scales is proposed.

\section{Development of a Constrained-IRT method for thermal measurements}

Full-field kinematic and thermal measurements are simultaneously performed during mechanical test using the experimental setup shown in Figure 1.

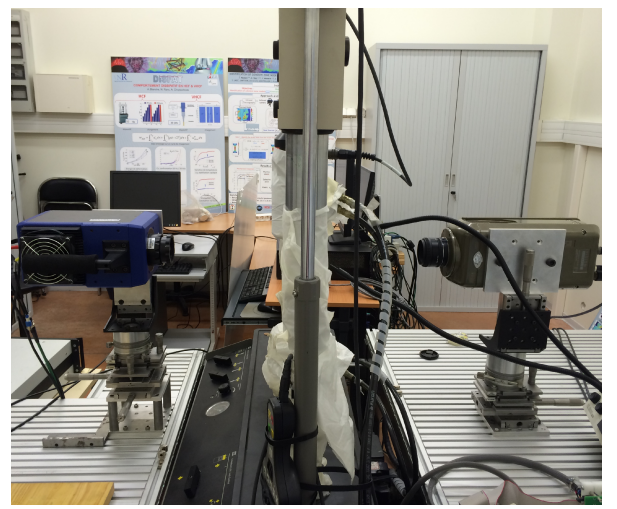

Figure 1. Experimental setup for fully coupled thermomechanical measurements

Thin flat samples with "millimetric" grains are used in this investigations. Preliminary metallurgical etching were performed on several specimens. They showed that the microstructure was very similar on both sides of the sample, which suggested that the sample had only one grain through the thickness. The microstructure was also carefully characterised through EBSD analysis in order to get the grain boundaries without perturbing the materials.

During the test, the sample is observed on one side with a CCD camera to perform DIC for displacement field measurements, while the opposite side is recorded at the same time with an IR camera to determine the surface temperature of the monitored area.

Spatiotemporal matching between the CCD and the IR coordinate systems is performed, as presented in $[17,18]$.

By construction, every imaging device records images in the Eulerian configuration. DIC algorithms are then applied to the visible images to obtain the kinematic transformation between the initial state and the current deformed ones. The IR camera records the thermal field of a deforming body in the Eulerian configuration during mechanical loading. Therefore, in order to monitor thermal variations at each material point on the monitored surface, the kinematic transformation obtained by DIC analysis is used to express the IR images in the Lagrangian configuration before computing the heat sources as described in $[6,14,15,19]$. 
Here, the kinematic measurements are obtained using the "Constrained-DIC" method described in [20]. This method allows to obtain kinematic fields consistent with restrictions imposed by the microstructure (e.g. intergranular slip, etc). The displacement fields are thus obtained on a mesh respecting the specimen microstructure (given here by the EBSD analysis on one side of the sample).

\subsection{From Eulerian to Lagrangian thermography}

Given the displacement fields per grain and the geometrical spatial matching between the CCD and the IR imaging systems [17], the thermal data provided by the IR camera are spatially interpolated in a linear manner (in space and in time) using the deformed configuration position given by the Constrained-DIC computation. This operation allows tracking of material particles associated with the DIC computational mesh, and thus enables us to associate the microscrutural orientation and grain boundaries to IR images in the Lagrangian configurations as presented in [17].

Since the IR camera records thermal radiations of the observed surface, a pixel calibration protocol described in [21] is applied to determine the temperature of the specimen induced by mechanical loading.

\subsection{Constraining the thermal description}

\subsubsection{Spatial discretization of the geometry}

A spatial discretization of the geometry is carefully performed in IR image in the Lagrangian configuration: an unstructured mesh is applied within each grain in order to keep the representation of the physical grain boundaries. The smallest mesh unit within each grain is called an "element", and each element consists of a set of IR pixels localized within the polygonal support of the element. The element boundaries are accurately determined and associated under specific adjacency conditions between elements.

Naturally it is possible for IRT analysis to use the same mesh as that for the DIC analysis or to use a coarser or a finer one. Taking into account the lower spatial resolution of the IR camera compared to the visible one, a coarser mesh is preferred here.

Figure 2 illustrates the computational mesh of the geometry for the Constrained-IRT analysis, which is used for the study in Section 3. The grain boundaries are in magenta and the element contours are plotted in black.

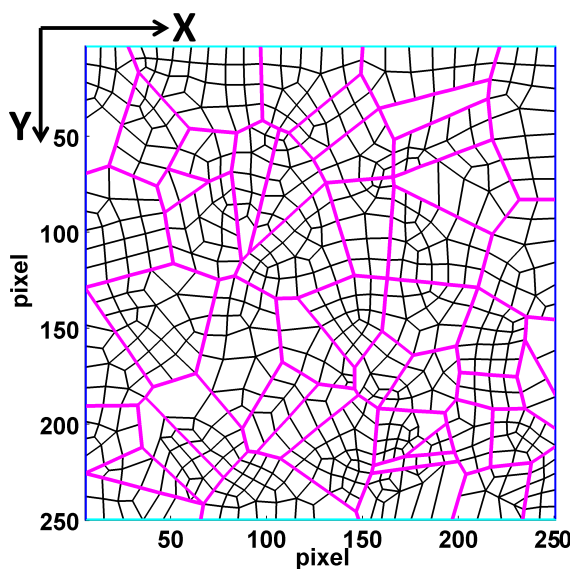

Figure 2. Computational mesh for IRT analysis 


\subsubsection{Thermal restriction constraint}

In order to precisely describe the local temperature variations, a thermal shape function (Equation 1) is assigned to each element of the mesh to represent the local temperature variations.

$$
\mathbf{T}\left(X, Y, t, p_{T}\right)=\sum_{m=0}^{d m} \sum_{n=0}^{d n} \sum_{l=0}^{d l} \alpha_{m n l} X^{m} Y^{n} t^{l}
$$

where, $(X, Y)$ represents the IR coordinates in the Lagrangian configuration, $t$ denotes the time and $p_{T}=\left(\alpha_{m n l}\right)$, is the parameter vector of the shape function. The temperature fields in each element are thus described in the real space, and not in a "reference element", as is the case for classical Finite Element (FE) descriptions.

This type of shape function includes constant polynomial (zero-order), multi-linear polynomial (first-order) functions and can be extended to quadratic, bi-quadratic polynomial (second-order) and possibly higher-order polynomial functions. The order and the type of the shape function can be chosen according to requirements. For instance,

- $d l=0$, represents an exclusively spatial shape function.

- $d m=d n=d$, represents a "symmetrical" shape function in $X$ and $Y$ direction.

- $d m=d n=d$ and $m+n \leqslant d$, represents a $d$-order shape function.

In order to account for an highly complex thermal fields, it is possible to use high order shape functions, with the drawbacks of increased CPU time and possibly less effective noise filtering.

Once the shape function is chosen, different thermal constraints can be introduced to describe the different thermomechanical features of the material at the observation scale. Figure 3 illustrates the situation between two adjacent elements $i$ and $j$.

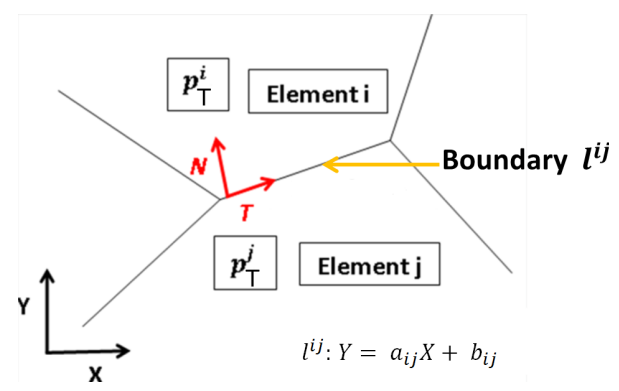

Figure 3. Description of the boundary $l^{i j}$ between two adjacent elements $i$ and $j$ and definition of the local Normal-Tangential coordinate system $\left(\vec{N}^{i j}, \vec{T}^{i j}\right)$ of the boundary $l^{i j}$.

- The shape function for element $i$ is:

$$
\mathbf{T}^{i}\left(X, Y, t, p_{T}^{i}\right)=\sum_{m=0}^{d m^{i}} \sum_{n=0}^{d n^{i}} \sum_{l=0}^{d l^{i}} \alpha_{m n l}^{i} X^{m} Y^{n} t^{l}
$$


- The shape function for element $j$ is:

$$
\mathbf{T}^{j}\left(X, Y, t, p_{T}^{j}\right)=\sum_{m=0}^{d m^{j}} \sum_{n=0}^{d n^{j}} \sum_{l=0}^{d l^{j}} \alpha_{m n l}^{j} X^{m} Y^{n} t^{l}
$$

- The boundary $l^{i j}$ between elements $i$ and $j$ is modelled as a linear relation between $X$ and $Y$. For a relatively "horizontal" boundary, as shown in Figure 3, the boundary $l^{i j}$ is expressed as $Y=a_{i j} X+b_{i j}$. Naturally, the case of a "vertical" boundary is deduced by inverting the role of $X$ and $Y$, expressed as $X=a_{i j}^{\prime} Y+b_{i j}^{\prime}$.

The coefficients $\left\{a_{i j}, b_{i j}\right\}$ or $\left\{a_{i j}^{\prime}, b_{i j}^{\prime}\right\}$ of the boundary expression are defined by the geometrical mesh. The normal and tangential vector $\vec{N}^{i j}$ and $\vec{T}^{i j}$ of the boundary $l^{i j}$ are deduced from $a_{i j}$ or $a_{i j}^{\prime}$. Furthermore, the degrees of the polynomials for thermal description $\left\{d m^{i}, d n^{i}, d l^{i}\right\}$ and $\left\{d m^{j}, d n^{j}, d l^{j}\right\}$ are not necessarily identical.

The restriction conditions can be introduced along a given boundary $l^{i j}$ between two adjacent elements $i$ and $j$, as shown in Figure 3, with the local Normal-Tangential coordinate system $\left(\vec{N}^{i j}, \vec{T}^{i j}\right)$ of the boundary $l_{i j}$. In this paper, only continuity restriction are addressed, namely:

\section{- Continuity of the temperature field}

The continuity of the temperature field is expected in continuous media, while temperature jumps can be observed in cracked specimen. The continuity of the thermal field can be introduced on the boundary $l^{i j}$ with the following restriction equation:

$$
\mathbf{T}^{i}\left(X, Y, t, p_{T}^{i}\right)=\mathbf{T}^{j}\left(X, Y, t, p_{T}^{j}\right) \quad \forall(X, Y) \in l^{i j}
$$

By introducing Equations 2 and 3 into Equation 4, we obtain the following expression:

$$
\sum_{m=0}^{d m^{i}} \sum_{n=0}^{d n^{i}} \sum_{l=0}^{d l^{i}} \alpha_{m n l}^{i} X^{m} Y^{n} t^{l}-\sum_{m=0}^{d m^{j}} \sum_{n=0}^{d n^{j}} \sum_{l=0}^{d l^{j}} \alpha_{m n l}^{j} X^{m} Y^{n} t^{l}=0
$$

When introducing the expression of the boundary $l^{i j}$ and using the classical binomial expansion: $Y^{n}=\left(a_{i j} X+b_{i j}\right)^{n}=\sum_{k=0}^{n} \mathbf{C}_{n}^{k}\left(a_{i j} X\right)^{k}\left(b_{i j}\right)^{n-k}$ with $\mathbf{C}_{n}^{k}=\frac{n !}{k !(n-k) !}$, we obtain the final restriction equation for the continuity of the temperature field:

$$
\begin{aligned}
\sum_{m=0}^{d m^{i}} \sum_{n=0}^{d n^{i}} \sum_{l=0}^{d l^{i}} \sum_{k=0}^{d n^{i}} \alpha_{m n l}^{i} \mathbf{C}_{n}^{k} a_{i j}^{k} b_{i j}^{n-k} & X^{m+k} t^{l} \\
& -\sum_{m=0}^{d m^{j}} \sum_{n=0}^{d n^{j}} \sum_{l=0}^{d l^{j}} \sum_{k=0}^{d n^{j}} \alpha_{m n l}^{j} \mathbf{C}_{n}^{k} a_{i j}^{k} b_{i j}^{n-k} X^{m+k} t^{l}=0
\end{aligned}
$$

Equation 6 corresponds to a polynomial expression in $X$ and $t$, with the degree equal to $\mathbf{S}^{i j}=\max \left\{d m^{i}+d n^{i}, d m^{j}+d n^{j}\right\}$ in $X$ and $\mathbf{L}^{i j}=\max \left\{d l^{i}, d l^{j}\right\}$ in $t$. This 
polynomial is null if and only if each of its coefficients is equal to zero, which gives $\left(1+\mathbf{S}^{i j}\right) \times\left(1+\mathbf{L}^{i j}\right)$ linear equations in $\alpha_{m n l}^{i}$ and $\alpha_{m n l}^{j}$, whose coefficients depend solely on the geometry through $a_{i j}$ and $b_{i j}$.

\section{- Continuity of temperature gradient}

In the same manner, restriction conditions can also be imposed on the temperature gradient at the elements boundaries. For homogeneous materials following Fourier's conduction law, the continuity of the heat flux is equivalent to the continuity of the temperature gradient, while it is related to a ratio in the temperature gradients proportional to the ratio of the different thermal conductivities for heterogeneous materials.

The continuity of the temperature gradient can be imposed at boundary $l^{i j}$ by introducing the following restrictions on the temperature spatial derivatives:

$$
\vec{\nabla} \mathbf{T}^{i}=\vec{\nabla} \mathbf{T}^{j} \Longleftrightarrow\left(\begin{array}{c}
\frac{\partial \mathbf{T}^{i}}{\partial X} \\
\frac{\partial \mathbf{T}^{i}}{\partial Y}
\end{array}\right)_{X, Y}=\left(\begin{array}{c}
\frac{\partial \mathbf{T}^{j}}{\partial X} \\
\frac{\partial \mathbf{T}^{j}}{\partial Y}
\end{array}\right)_{X, Y} \forall(X, Y) \in l^{i j}
$$

As previously, the temperature spatial derivatives can be expressed as:

$$
\begin{gathered}
\text { element } i\left\{\begin{array}{l}
\frac{\partial \mathbf{T}^{i}}{\partial X}=\sum_{m=1}^{d m^{i}} \sum_{n=0}^{d n^{i}} \sum_{l=0}^{d l^{i}} \sum_{k=0}^{d n^{i}} m \alpha_{m n l}^{i} \mathbf{C}_{n}^{k} a_{i j}^{k} b_{i j}^{n-k} X^{m+k-1} t^{l} \\
\frac{\partial \mathbf{T}^{i}}{\partial Y}=\sum_{m=0}^{d m^{i}} \sum_{n=1}^{d n^{i}} \sum_{l=0}^{d l^{i}} \sum_{k=0}^{d n^{i}-1} n \alpha_{m n l}^{i} \mathbf{C}_{n-1}^{k} a_{i j}^{k} b_{i j}^{n-k-1} X^{m+k} t^{l}
\end{array}\right. \\
\text { element } j\left\{\begin{array}{l}
\frac{\partial \mathbf{T}^{j}}{\partial X}=\sum_{m=1}^{d m^{j}} \sum_{n=0}^{d n^{j}} \sum_{l=0}^{d l^{j}} \sum_{k=0}^{d n^{j}} m \alpha_{m n l}^{j} \mathbf{C}_{n}^{k} a_{i j}^{k} b_{i j}^{n-k} X^{m+k-1} t^{l} \\
\frac{\partial \mathbf{T}^{j}}{\partial Y}=\sum_{m=0}^{d m^{j}} \sum_{n=1}^{d n^{j}} \sum_{l=0}^{d l^{j}} \sum_{k=0}^{d n^{j}-1} n \alpha_{m n l}^{j} \mathbf{C}_{n-1}^{k} a_{i j}^{k} b_{i j}^{n-k-1} X^{m+k} t^{l}
\end{array}\right.
\end{gathered}
$$

The continuity of the temperature gradient on the boundary $l^{i j}$ leads to two sets of polynomial equations in $X$ and $t$ (for a relatively "horizontal" boundary) : one for the $X$-derivative (eq. 8.a and 9.a) and one for the $Y$-derivative (eq. 8.b and 9.b). The degree of these polynomials are equal to $\mathbf{S}^{i j}=\max \left\{d m^{i}+d n^{i}, d m^{j}+d n^{j}\right\}$ in $X$ and $\mathbf{L}^{i j}=\max \left\{d l^{i}, d l^{j}\right\}$ in $t$.

Like Equation 6 for the temperature continuity, this expression also gives $(1+$ $\left.\mathbf{S}^{i j}\right) \times\left(1+\mathbf{L}^{i j}\right)$ linear equations in $\alpha_{m n l}^{i}$ and $\alpha_{m n l}^{j}$, with the coefficients depending only on $a_{i j}$ and $b_{i j}$.

It can be interesting to express the continuity conditions in the local frame $\left(\vec{N}^{i j}, \vec{T}^{i j}\right)$ associated with the boundary $l^{i j}$ rather than in the global Cartesian coordinate system $(\vec{X}, \vec{Y})$. For thermally isotropic materials, the continuity of the normal (resp. tangential) heat flux leads to the following polynomial expression (with $\vec{A}=\vec{N}^{i j}$, or $\vec{A}=\vec{T}^{i j}$ ): 


$$
\begin{aligned}
A_{X} \sum_{m=1}^{d m^{i}} \sum_{n=0}^{d n^{i}} \sum_{l=0}^{d l^{i}} \sum_{k=0}^{d n^{i}} m \alpha_{m n l}^{i} \mathbf{C}_{n}^{k} a_{i j}^{k} b_{i j}^{n-k} X^{m+k-1} t^{l} \\
+A_{Y} \sum_{m=0}^{d m^{i}} \sum_{n=1}^{d n^{i}} \sum_{l=0}^{d l^{i}} \sum_{k=0}^{d n^{i}-1} n \alpha_{m n l}^{i} \mathbf{C}_{n-1}^{k} a_{i j}^{k} b_{i j}^{n-k-1} X^{m+k} t^{l} \\
-A_{X} \sum_{m=1}^{d m^{j}} \sum_{n=0}^{d n^{j}} \sum_{l=0}^{d l^{j}} \sum_{k=0}^{d n^{j}} m \alpha_{m n l}^{j} \mathbf{C}_{n}^{k} a_{i j}^{k} b_{i j}^{n-k} X^{m+k-1} t^{l} \\
-A_{Y} \sum_{m=0}^{d m^{j}} \sum_{n=1}^{d n^{j}} \sum_{l=0}^{d l^{j}} \sum_{k=0}^{d n^{j}-1} n \alpha_{m n l}^{j} \mathbf{C}_{n-1}^{k} a_{i j}^{k} b_{i j}^{n-k-1} X^{m+k} t^{l}=0
\end{aligned}
$$

Naturally, for thermally anisotropic materials, the continuity of the heat flux at the boundary requires the introduction of supplementary terms involving the ratio of the in-plane heat diffusion coefficients.

The three above constraint conditions can be used together or independently to describe the different thermal features of the transformation: (continuous/discontinuous) (temperature and/or temperature gradient). For example, a perfect interface is related to continuous temperature and heat flux, while the development of a crack leads to a discontinuity of thermal field with a null heat flux (provided conduction and radiation losses are neglected on the boundary). Finally, the thermal behavior of a real grain boundary is not well understood, as its mechanical behavior. Consequently, a simple way to model such a boundary is to consider it as an additional body introducing a thermal resistance (associated with a continuous heat flux and a discontinuous temperature).

Taking into account these different constraints leads to imposing the corresponding linear equations between the two elements $i$ and $j$, which leads to the following linear system:

$$
\left[\mathbf{A}_{\mathbf{T}}^{\mathbf{i j}}\right]\left\{\mathbf{P}_{\mathbf{T}}^{\mathbf{i j}}\right\}=\{\mathbf{0}\}
$$

where $\mathbf{A}_{\mathbf{T}}^{\mathbf{i j}}$ is the elementary restriction matrix between element $i$ and $j$, and $\mathbf{P}_{\mathbf{T}}^{\mathbf{i j}}$ is the elementary vector containing all the unknown parameters $\left(p_{T}^{i}\right.$ and $\left.p_{T}^{j}\right)$ of the thermal shape functions for the two elements $i$ and $j$.

By iterating this operation for all boundaries on which continuity constraints are applied, it is possible to assemble a global restriction matrix $\left(\mathbf{A}_{\mathbf{T}}\right)$ for all elements of the computational mesh on which restrictions are applied, as well as a global vector $\left(\mathbf{P}_{\mathbf{T}}\right)$ containing all the thermal parameters for these elements.

$$
\left[\mathbf{A}_{\mathbf{T}}\right]\left\{\mathbf{P}_{\mathbf{T}}\right\}=\{\mathbf{0}\}
$$

Moreover, it is important to recall that, since we work in the Lagrangian framework, all restriction matrices are determined in the initial configuration, so they have to be computed only once throughout the data processing.

By construction, inequality constraints could also be imposed to account for an inter- 
granular crack formed in polycrystalline aggregates. In addition, instead of the constraint imposed upon all the boundary points, it is possible to impose it on a limited number of nodes on the element boundary in order to obtain less constraint equations and thus a more compliant thermal description.

\subsection{Solving the enriched thermal problem}

\subsubsection{Numerical resolution}

As mentioned in Section 2.1, the measured temperature fields are obtained using "Lagrangian thermography". In order to filter the noise on the temperature measurements $\left(\mathbf{T}^{e x p}\right)$, the temperature field is projected on the chosen shape functions using a least square method. This operation can be formulated as:

$$
\mathfrak{C}_{T}=\sum_{\mathbf{e}} \sum_{i_{e}} \sum_{l}\left[\mathbf{T}^{\mathbf{e}}\left(X_{i_{e}}, Y_{i_{e}}, t_{l}, p_{T}^{\mathbf{e}}\right)-\mathbf{T}^{\exp }\left(X_{i_{e}}, Y_{i_{e}}, t_{l}\right)\right]^{2} \Delta X \Delta Y
$$

The summation on e spans all the elements of the domain, while the one on $i_{e}$ involves all the IR pixels inside of each element $\mathbf{e}$. With the shape functions $\mathbf{T}^{\mathbf{e}}$ being time-dependent, a time smoothing is also performed by taking into account $l$ time steps in the computation of the gap $\mathfrak{C}_{T}$. The quantities $\Delta X$ and $\Delta Y$ represent the pixel size.

The minimisation of the $\mathfrak{C}_{T}$ is performed under the constraints associated with the previously mentioned thermal restrictions described in Equation 12:

$$
\begin{gathered}
\mathbf{P}_{\mathbf{T}}^{\text {opt }}=\underset{p_{T}}{\operatorname{argmin}}\left\{\mathfrak{C}_{T}\right\} \\
\text { with }\left[\mathbf{A}_{\mathbf{T}}\right]\left\{\mathbf{P}_{\mathbf{T}}\right\}=\{\mathbf{0}\}
\end{gathered}
$$

The matrix $\mathbf{A}_{\mathbf{T}}$ is not invertible: the linear system in Equation 12 has necessarily a non-trivial solution corresponding to the situation where the shape functions are identical ("homogeneous" solution). Consequently, the rank $(\gamma)$ of the matrix $\mathbf{A}_{\mathbf{T}}$ is smaller than the number of unknowns $(\eta)$ in vector $\mathbf{P}_{\mathbf{T}}$.

Let $\mathbf{A}_{\mathbf{T}}^{\text {tri }}$ be the matrix after the triangulation:

$$
\left[\mathbf{A}_{\mathbf{T}}^{\mathrm{tri}}\right]=\left[\mathbf{A}_{\mathbf{T}_{1}} \mid \mathbf{A}_{\mathbf{T}_{2}}\right]
$$

where, $\mathbf{A}_{\mathbf{T}_{\mathbf{1}}}$ is an invertible square matrix with $\gamma$ lines. The matrix $\mathbf{A}_{\mathbf{T}_{\mathbf{2}}}$ has $\gamma$ lines and $\eta-\gamma$ columns.

The linear system in Equation 12 can be reformulated as:

$$
\left[\mathbf{A}_{\mathbf{T}_{1}} \mid \mathbf{A}_{\mathbf{T}_{2}}\right]\left\{\begin{array}{l}
\mathbf{P}_{\mathbf{T}_{1}} \\
\mathbf{P}_{\mathbf{T}_{2}}
\end{array}\right\}=\{\mathbf{0}\} \quad \Longleftrightarrow \mathbf{P}_{\mathbf{T}_{1}}=\underbrace{-\mathbf{A}_{\mathbf{T}_{1}}^{-1} \mathbf{A}_{\mathbf{T}_{2}}}_{\mathbf{A}_{\mathbf{e q}}} \mathbf{P}_{\mathbf{T}_{2}}
$$

where, $\mathbf{P}_{\mathbf{T}_{\mathbf{2}}}$ represents the "independent" parameters to be used to describe the temperature fields, and $\mathbf{P}_{\mathbf{T}_{1}}$ stands for the dependent parameters deduced from the thermal constraints (Equation 16). The constrained linear least-square minimization consists in finding the optimal set of "independent" parameters verifying: 


$$
\begin{gathered}
\mathbf{P}_{\mathbf{T}_{2}}^{\text {opt }}=\underset{\mathbf{P}_{\mathbf{T}_{2}}}{\operatorname{argmin}}\left\{\mathfrak{C}_{T}\left(\begin{array}{c}
\mathbf{A}_{\mathbf{e q}} \mathbf{P}_{\mathbf{T}_{\mathbf{2}}} \\
\mathbf{P}_{\mathbf{T}_{2}}
\end{array}\right)\right\} \\
\text { with } \mathbf{P}_{\mathbf{T}_{1}}^{\text {opt }}=\mathbf{A}_{\mathbf{e q}} \mathbf{P}_{\mathbf{T}_{\mathbf{2}}}^{\text {opt }}
\end{gathered}
$$

\subsubsection{Heat source computation}

Once the parameters $\mathbf{P}_{\mathbf{T}}^{\text {opt }}$ have been determined, the thermal shape function $\mathbf{T}$ can be analytically differentiated in order to compute the time derivative and the Laplacian of the temperature in order to compute the grain-scale local heat source field. We then compute the heat sources following the method described in $[5,6,19,22-24]$ :

$$
\frac{w_{c h}^{\prime}}{\rho C}=\frac{\mathrm{d} \theta}{\mathrm{d} t}+\frac{\theta}{\tau^{2 D}}-\frac{k}{\rho C}\left(\frac{\partial^{2} \theta}{\partial X^{2}}+\frac{\partial^{2} \theta}{\partial Y^{2}}\right)
$$

where $\theta(X, Y, t)$ represents the temperature variation between the initial and the current state in space and in time.

To summarize, using this Constrained IR data processing method, different microstructural thermal hypotheses can be introduced in the description of the temperature field. For instance,

- In the least constrained configuration, no continuity constraints at all are imposed a priori between adjacent elements. This would be suitable for describing any situation (perfect or imperfect conduction due to cracking or thermal resistances at the boundary, ...).

- The most constrained situation is associated with the enforcing of thermal continuity (temperature and temperature gradient) at the boundaries of all elements. This situation amounts to imposing perfect intergranular interfaces. In general, this configuration is not compliant enough to represent complex thermal fields.

- The intermediate situation consists of imposing continuity restrictions on the boundaries of all elements belonging to a given grain. The thermal field is thus continuous within each grain but possibly discontinuous between two adjacent grains. This configuration is particularly relevant to describe intragranular thermal heterogeneities with perfect intragranular boundaries and intergranular imperfect boundaries, without any a priori assumption on the thermal behavior of the imperfect boundary. Post-processing of the thermal field at the grain boundaries can then be used to get information on the interface response.

In the following paper, the performance of this Constrained IR data processing method will be demonstrated through a numerical example on an aluminum polycrystalline aggregates.

\section{Numerical validation}

\subsection{Numerical thermal image generation}

To validate the proposed method on heterogeneous thermal fields, we used computergenerated thermal images associated with a completely known heat source field. In order to obtain a heat source field compatible with that obtained during straining of an alu- 


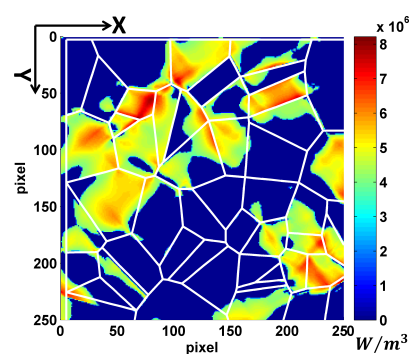

(a) Local theoretical heat source field, $w_{c h}^{\prime t h}$

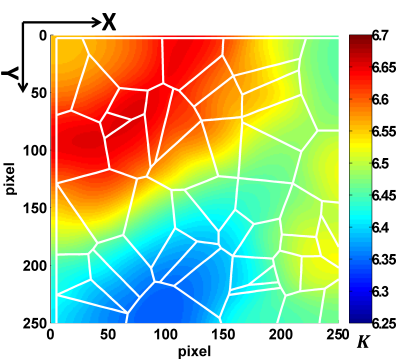

(b) Theoretical temperature variation field, $\theta^{\text {th }}$

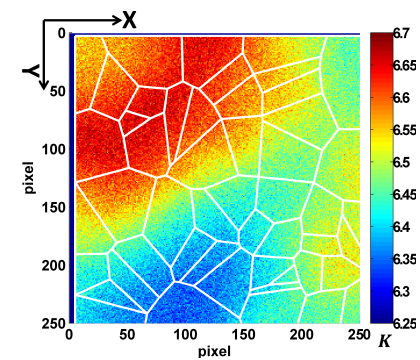

(c) Noisy temperature variation field, $\theta^{\text {noisy }}$

Figure 4. Theoretical heat source field for COMSOL Multiphysics and the theoretical and the noisy temperature variation field.

Table 1. Table of the material thermophysical parameters

\begin{tabular}{cccc}
\hline$\rho\left(k g / \mathrm{m}^{3}\right)$ & $C(\mathrm{~J} / \mathrm{kg} / \mathrm{K})$ & $k(W / \mathrm{m} / \mathrm{K})$ & $T_{0}^{\text {ref }}(\mathrm{K})$ \\
\hline 2700 & 950 & 260 & 300 \\
\hline
\end{tabular}

minum polycrystal, we opted to define the heat source field as a function of the strain-rate field obtained by Finite Element (FE) simulation on a given microstructure using a crystal plasticity law. FE simulation was performed using Code Aster with the Meric-Cailletaud crystal plasticity law $[25,26]$ for FCC slip systems and boundary conditions corresponding to uniform tension. The grain orientations of the microstructure are randomly chosen to obtain an isotropic effective tensile response that roughly corresponds to that of an aluminium alloy with the chosen set of parameters [20].

For the kinematic numerical study, a realistic aggregate of 50 millimetric grains was generated using conventional Voronoï tessellation, which provides convex polyhedric grains and a microstructure representative of that of aluminium or austenitic stainless steels. This experimental tensile test simulation was performed using a mesh of 113000 quadratic triangular elements in a bi-dimensional framework under a plane strain assumption.

The aim of this FE simulation is to provide realistic local kinematic fields associated with equilibrated stress fields and corresponding to realistic strain and stress heterogeneities. The simulated strain rate fields were then used to generate a heat source field compatible with the development of localised plasticity within the grains, as shown in Figure 4(a), where the white lines represent the physical grain boundaries. Naturally, the generated heat sources were not meant to respect the energy balance of the real material during loading.

The strain-rate variations are exported from Code Aster and then imported in COMSOL Multiphysics. A pure thermal computation is then performed assuming homogeneous thermal properties of the specimen and perfect thermal interfaces between each grain (see Table 1). This time-dependent problem is solved in two dimensions. As shown in Figure 2 , the thermal boundary conditions correspond to convection exchanges on the left and right boundaries ( $h_{\text {contour }}$ in blue color), and the top and bottom boundaries ( $h_{\text {grip }}$ in cyan color). Heat losses by convection are also taken into account on the specimen surface ( $\left.h_{\text {surface }}\right)$, as shown in Table 2.

The initial temperature is supposed to be homogeneous over the whole specimen surface. The thermal interfaces between two a adjacent grains are perfect, i.e. continuous temperature and heat flux. In order to mimic the acquisition of a series of images by an IR camera, the computed thermal field is sampled at $100 \mathrm{~Hz}$ frequency on a regular 256 pixel $\times 256$ pixel grid corresponding to that of a commercially available IR sensor, as 


\begin{tabular}{|c|c|c|}
\hline$h_{\text {contour }}$ & $h_{\text {grip }}$ & $h_{\text {surface }}$ \\
\hline 25 & 100 & 25 \\
\hline
\end{tabular}

presented in Figure 4(b). The physical duration of the simulation is $6 s$, which is consistent with real testing conditions.

Supplementary noise (white, centered, Gaussian with a $0.025 K$ standard deviation) is added to mimic the thermal noise of the IR camera, shown in 4(c). This noise distribution is consistent with the metrology assessments of commercially available IR cameras.

The thermal fields are computed using a purely thermal simulation, so the geometry of the specimen is kept constant throughout the simulation. The temperature fields are thus obtained in the initial reference configuration, which directly gives the Lagrangian temperature maps. In order to get the Eulerian temperature maps, corresponding to those actually measured by IR cameras, it is possible to deform these temperature maps with displacement fields associated with the kinematic simulation.

In order to focus only on the thermal aspects of the data processing in this investigation, we exclusively use in this paper the Lagrangian temperature fields, thus assuming that the displacement field is perfectly known on the specimen surface. Naturally, errors in the displacement field measurements generate supplementary uncertainties in the heat source estimation, which are not studied in this paper.

\subsection{Spatial discretization of the synthetic image}

As already mentioned in section 2.2.1, the geometry is meshed (here using "free" quadrangular elements). Note that the mesh respects the geometry of the microstructure used for the kinematic simulation. This mesh is thus consistent with the imposed heat source distribution. The magenta color represents the physical grain boundaries (see Figure 2).

Each element has an identical shape function which is bi-quadratic in space and linear in time, as shown in Equation 1, with $d m=d n=2$ and $d l=1$. Consequently, the parameter vector $p_{T}$ has 18 components for each element.

\subsection{Numerical results}

The data processing applied here involves a "total" continuity (temperature and temperature gradient) for all the adjacent elements within each grain, and no continuity relation on the grain boundaries:

- Continuity on temperature and on the normal and tangential temperature gradients

- A time smoothing "window" of 60 IR images.

The 600 noisy IR temperature variation maps (100 $\mathrm{Hz}$ during $6 \mathrm{~s}$ ) are then processed with the parameters outlined above.

\subsubsection{Spatial variation patterns}

In this section, the computed thermal results (temperature and heat sources) are presented, along with the errors between computed and imposed data.

For instance, the noisy temperature variation fields illustrated in Figure 4(c) are taken as inputs of the procedure. The fitted temperature variation map shown in Figure 5(a) is 


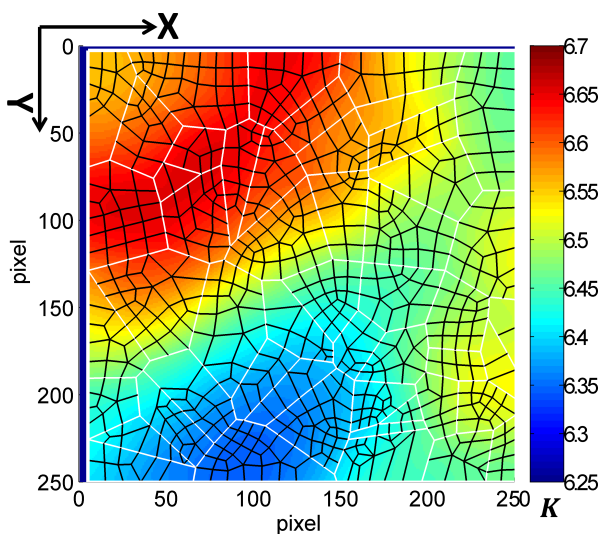

(a) The fitted temperature variation field, $\theta^{\text {fit }}$

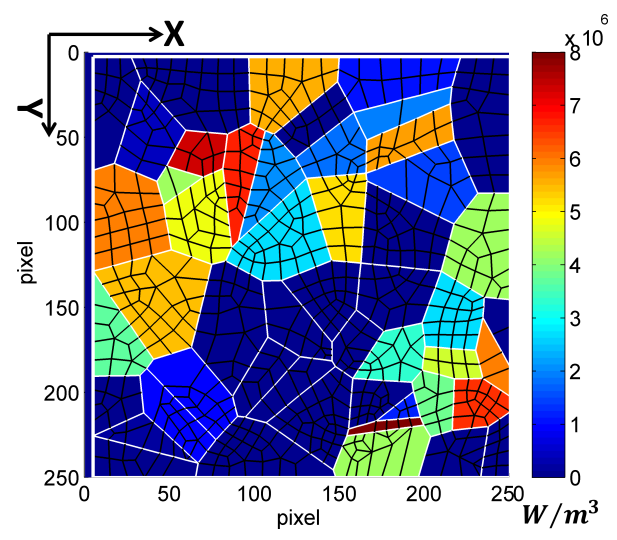

(b) The calculated heat source field, $w_{c h}^{\prime}$ fit

Figure 5. The calculated temperature variation field and the corresponding calculated grain averaged heat source field

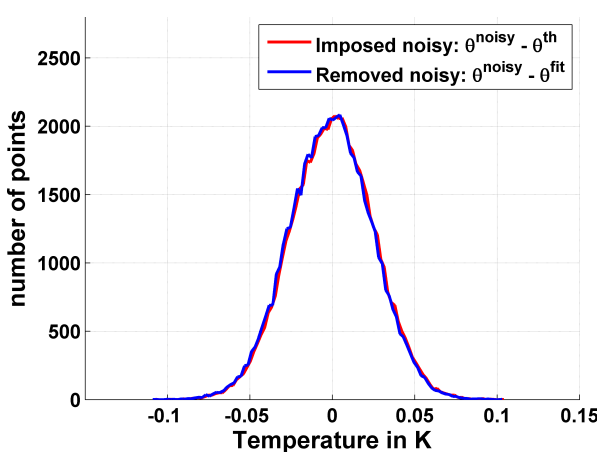

(a) The histogram of the imposed noise and the histogram of the removed noise by the Constrained IR method.

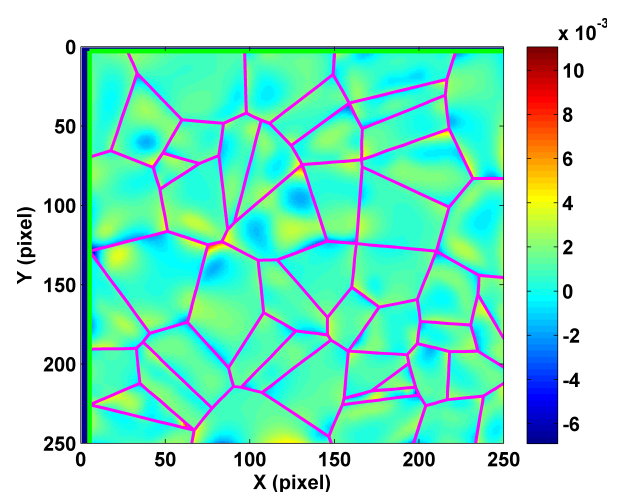

(b) The difference between the $\theta^{\text {fit }}$ and $\theta^{\text {th }}$

Figure 6. The histogram and the residual of the error for the temperature variation field estimation.

obtained by the Constrained IR data processing method. The corresponding heat source field could then be deduced, as presented in Figure 5(b).

Naturally it is important to quantify this data processing error. The error is defined by the the difference between the calculated results (temperature and heat sources) and their imposed "theoretical" values. Concerning the temperature variation field calculation, the residual between the measured noisy temperature variation field and the fitted one corresponds to the "noise" removed by the constrained data filtering. It is interesting to note that, in Figure 6(a), this residual has the same histogram as the imposed noise, which suggests that only the imposed noise was suppressed by the data processing procedure without loss of information. In addition, Figure 6(b) shows the difference between the fitted temperature variation $\theta^{\text {fit }}$ and the imposed theoretical variation $\theta^{\text {th }}$, which remains negligible (less than $0.01 \mathrm{~K}$ ) and is mainly localized near the grain boundaries, while the Standard Deviation (SD) of this difference is equal to $9 \cdot 10^{-4} \mathrm{~K}$. The localization of the temperature error on the grain boundaries has two concurring reasons. The first one is inherent to the fact that fitting regularly-spaced data gives less influence to the data closer to the boundaries compared to the ones in the centre. The second one is due to the fact that here, heat sources gradients are mainly localized in the vicinity of the grain boundaries which entails strong temperature gradients in these zones that are hardly accounted for by the chosen shape functions. 


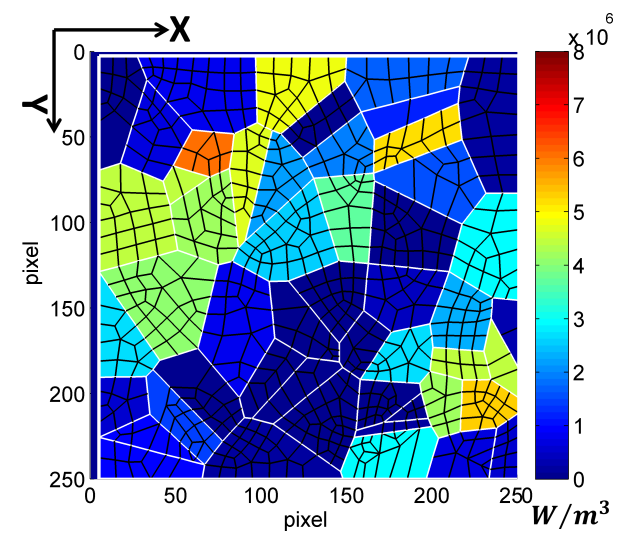

(a) The theoretical grain averaged heat source field, $w_{c h}^{\prime t h}$

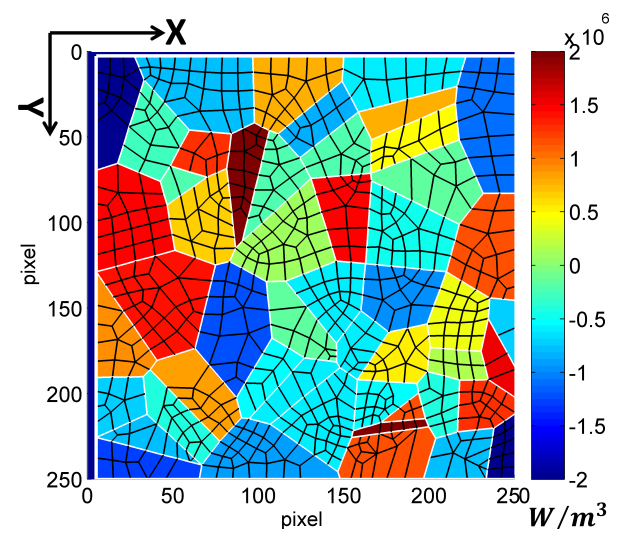

(b) The difference between the calculated and the theoretical heat source field.

Figure 7. The theoretical heat source field and the error for the heat source field estimation.

Using a temperature variation field calculated per element, it is possible to compare the averaged fields (temperature, heat sources) either per element or per grain.

As mentioned at the beginning of Section 3.3, an intragranular continuity restriction was imposed on temperature and on temperature gradients for each grain, i.e. the thermal field (temperature and temperature gradients) is rigorously continuous on the boundaries of all elements belonging to each grain. Consequently, a feeble heat source variation within each grain was obtained due to these strong spatial restrictions. Consequently, we present and compare the (measured and theoretical) heat source field at the grain scale. The theoretical heat source field is imported from the thermal simulation. It is presented in Figure 4(a). Its grain-averaged representation is shown in Figure 7(a). Figure 5(b) shows the "measured" grain-averaged heat sources distribution given by the Constrained IR data processing method. Finally, the measurement error is defined as the difference between "measured" (fitted) and "theoretical" heat sources (respectively $w_{c h}^{\prime} f i t$ and $w_{c h}^{\prime t h}$ ). It is illustrated in Figure 7(b) (grain-averaged error). The heterogeneity of the grain heat source response is obviously observed and quantitatively obtained, despite the high diffusivity of the material. It is important to notice that the error is centred (zero mean value), meaning that the measurement is unbiased. The amplitude of the error can be assessed through the standard deviation of the difference between $w_{c h}^{\prime f i t}$ and $w_{c h}^{\prime t h}$. It is worth $10^{6} \mathrm{~W} / \mathrm{m}^{3}$. This value is an order of magnitude smaller than the theoretical heat sources. Furthermore, the error is maximum in small and stretched grains (for which the fitting efficiency is the worst as they involve the identification of large sets of "independent" parameters for relatively small databases).

\subsubsection{Temporal variation patterns}

The processing method naturally allows to monitor the temporal evolution of temperature variation and heat source for each grain throughout mechanical loading.

Among the aggregates of 50 grains, 3 grains were chosen to illustrate the temporal variations in the thermal quantities. Grain 2 was chosen for its highest heat source value among the 50 grains, Grain 3 was chosen for the minimal heat source value, and an average one was selected as Grain 1.

The temporal variations in the imposed theoretical temperature $\theta^{\text {th }}$ and theoretical heat source $w_{c h}^{\prime t h}$ are separately plotted (using solid lines) in Figure 8 (a and b). After the Constrained IR data processing procedure, the fitted temperature variation $\theta^{f i t}$ and the calculated heat source $w_{c h}^{\prime f i t}$ are also respectively plotted (using dashed lines) per grain 


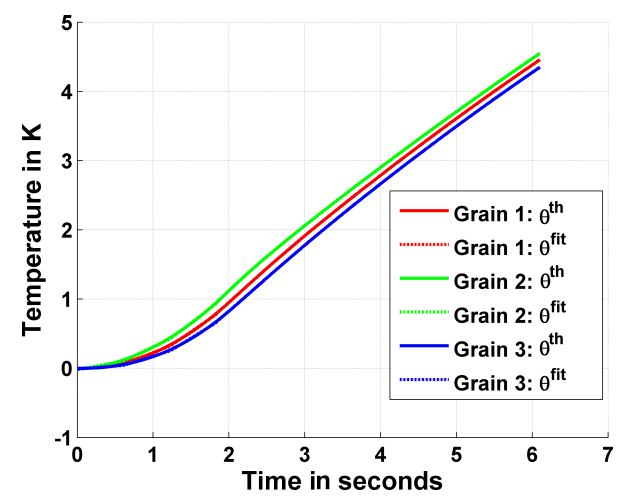

(a) Theoretical and fitted temperature variations per grain over time.

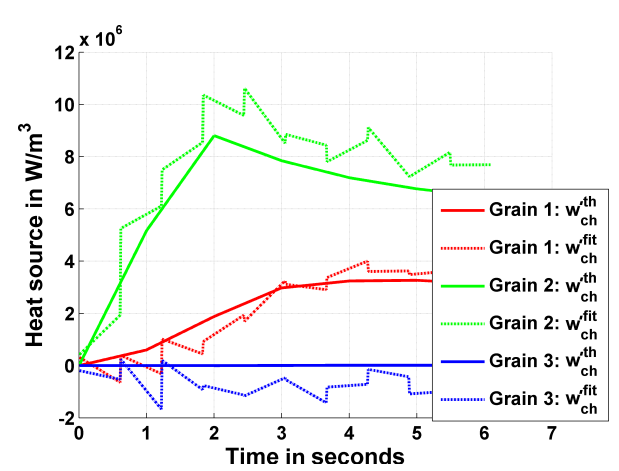

(b) Theoretical and calculated heat source variations per grain over time.

Figure 8. Temporal variations in the thermal data for 3 grains.

Table 3. The mean and standard deviation of the error between the calculated and the theoretical heat source for the 3 grains in $W / m^{3}$.

\begin{tabular}{lccc}
\hline & Grain 1 & Grain 2 & Grain 3 \\
\hline Mean & $-7.88 .10^{4}$ & $9.78 .10^{5}$ & $-6.88 .10^{5}$ \\
\hline Standard deviation & $4.72 .10^{5}$ & $6.23 .10^{5}$ & $3.55 .10^{5}$ \\
\hline
\end{tabular}

in Figure 8 ( $\mathrm{a}$ and $\mathrm{b}$ ). A very satisfactory agreement is obtained between the temporal variations in the theoretical and the fitted temperature for all 3 grains (Figure 8a), and the trend of each grain concerning the heat source response is also obtained and shown in Figure 8b. Naturally, the measured heat sources are piecewise continuous in time (here linear because the shape function is linear in time), and they present jumps at the end of each time-fitting window.

The temporal variations in the error $\theta^{\text {fit }}-\theta^{\text {th }}$ is presented in Figure 9(a), and the histogram of the hear sources error is shown in Figure 9(b). The temperature error (Figure $9(\mathrm{a})$ ) involves stepwise quadratic gaps (with a period equals $0.6 \mathrm{~s}$ ) induced by the choice of a linear shape function for the temporal description, which implicitly neglects the second order terms in the time course of the temperature variations. The use of second-order shape functions in time would then naturally lead to better results.

Concerning the error in the heat source estimation, as we can see in Figure 9(b), the three error histograms have almost the same standard deviation, but with a different average value for the 3 grains, as shown in Table 3 . The fact that these mean values are in the same order of magnitude as the standard deviations $\left(10^{5} \mathrm{~W} / \mathrm{m}^{3}\right)$, and they are ten times lower than the order of magnitude measured $\left(10^{6} \mathrm{~W} / \mathrm{m}^{3}\right)$ for the heat sources, indicates that the results provided by this data processing method can be trusted.

\section{Concluding comments}

In this paper, a specific thermal data processing method was developed to measure temperature and heat source fields in heterogeneous (polycristalline) materials. The method was validated on numerically-obtained data associated with heat diffusion in a polycristalline aluminum aggregates.

In order to perform this numerical validation, a kinematic FE simulation is conducted to provide local kinematic fields associated to the given microstructure. Assuming the heat source field is a function of the strain-rate field, a pure thermal computation is 


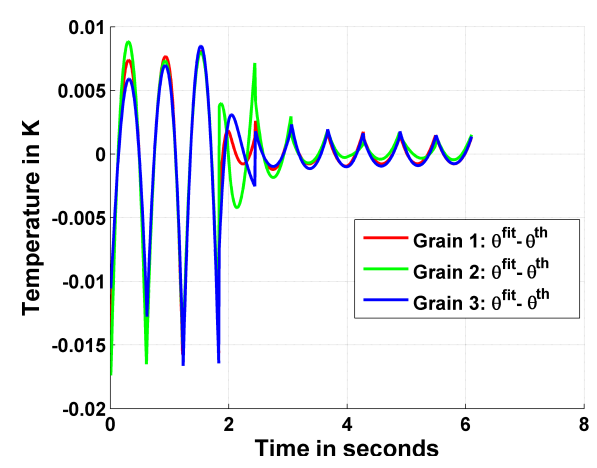

(a) Temporal variations in the error between the fitted and theoretical temperature variations par grain.

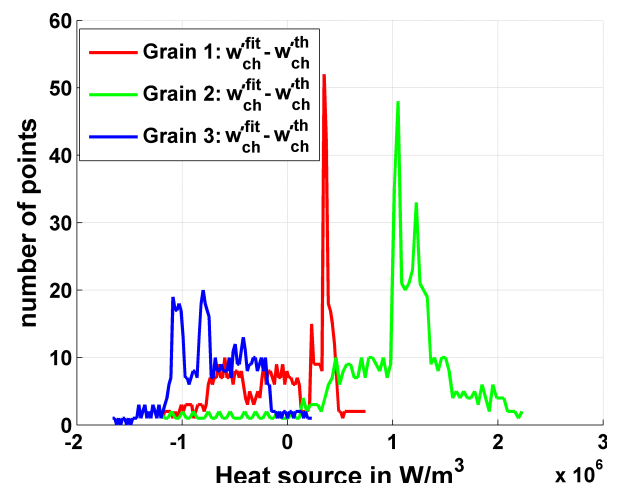

(b) Histograms of the error between the calculated and theoretical heat source for the 3 grains.

Figure 9. The thermal data processing error.

performed to obtain thermal fields associated with a completely known heat sources field. White Gaussian noise ( $0.025 \mathrm{~K}$ standard deviation) as encountered in standard IR cameras was added to the thermal images to mimic the real IR measurement condition. All of these synthetic thermal images were processed with the proposed Constrained IR data processing method. The data processing performance is illustrated using spatial and temporal comparison between the computed and imposed data (temperature and heat sources).

Through the results, a particularly effective denoising effect was demonstrated. From an imposed standard deviation of noise $(=0.025 K)$ to a standard deviation of noise $\left(=9 \cdot 10^{-4} \mathrm{~K}\right)$ in the fitted temperature fields, while maintaining a good description of the thermal gradient in time and space. Furthermore, the robustness of the proposed method with respect to superimposed noise should be investigated in further detail, notably taking into account the level of imposed restrictions. As already mentioned, the continuity constraints can be imposed in a weaker manner (e.g. continuity solely on the temperature or continuity on a limited set of nodes), in order to loosen the imposed constraints.

In this paper, the validation procedure of the proposed method is performed on a numerical example, which is associated with a complex situation, i.e. the imposed heat source fields are very heterogeneous with strong material diffusivity. However, the obtained results show a quantitatively correct estimate of measured heat sources $\left(10^{6} \mathrm{~W} / \mathrm{m}^{3}\right)$, with the mean and the standard deviation of error being in the order of magnitude of $10^{5}$ $W / m^{3}$. All of these results are conclusive to validate our methodology.

Naturally, several improvements could be proposed and tested later, such as:

- A higher order shape function could be used to obtain an accurate description of fine temperature gradients within each grain.

- A better temporal smoothing could be achieved using higher order polynomials and larger temporal fitting windows to improve the heat source measurement.

- Imposing continuity restriction in time in order to suppress the temperature jumps.

- Adjusting the thermal description, for example, thermal continuity can be imposed at the boundaries of all elements on a limited set of nodes to expect a better results.

By construction, all of these formalisms could be adapted to impose discontinuity constraints on the temperature field or on the thermal gradient field in order to account for crack development. Therefore, a similar investigation on a numerically cracked polycrystalline aggregate is currently achieved using the same methodology.

This Constrained IR data processing method can be naturally applied in future on 
experimental tests combining a local kinematic measurement method [20]. The coupling of these two measurements will allow to build the grain-scale energy balances in order to characterize and verify the thermomechanical consistency of some classical polycrystal plasticity models.

\section{References}

[1] B. Jaoul. Étude de la plasticité et application aux métaux. Dunod, 1965.

[2] W. Boas and M. E. Hargreaves. On the inhomogeneity of plastic deformation in the crystals of an aggregate. Proceedings of the Royal Society of London. Series A. Mathematical and Physical Sciences, 193(1032):89-97, 1948.

[3] W. S. Farren and G. I. Taylor. The heat developed during plastic extension of metals. Proceedings of the Royal Society of London. Series A, 107(743):422-451, 1925.

[4] G. I. Taylor and H. Quinney. The latent energy remaining in a metal after cold working. Proceedings of the Royal Society of London. Series A, 143(849):307-326, 1934.

[5] A. Chrysochoos and O. Maisonneuve. Bilan énergétique en élastoplasticité grandes déformations. Comptes rendus de l'Académie des sciences. Série 2, Mécanique, Physique, Chimie, Sciences de l'univers, Sciences de la Terre, 300(20):985-990, 1985. fre.

[6] A. Chrysochoos, B. Wattrisse, J.-M. Muracciole, and Y.EL. Kaim. Fields of stored energy associated with localiszed necking of steel. Mechanics of Materials and Structures, Volume 4, 2009.

[7] M.A. Sutton, W.J. Wolters, W.H. Peters, W.F. Ranson, and S.R. McNeill. Determination of displacements using an improved digital correlation method. Image and Vision Computing, 1(3):133 - 139, 1983.

[8] B. Pan, K.M. Qian, H.M. Xie, and A. Asundi. Two-dimensional digital image correlation for in-plane displacement and strain measurement: a review. Measurement Science and Technology, 20(6):062001, 2009.

[9] Brent L. Adams, Stuart I. Wright, and K. Kunze. Orientation imaging: The emergence of a new microscopy. Metallurgical Transactions A, 24:819-831, 1993.

[10] L. Delannay, O.V Mishin, D.Juu Jensen, and P. Van Houtte. Quantitative analysis of grain subdivision in cold rolled aluminium. Acta Materialia, 49(13):2441 - 2451, 2001.

[11] F.J. Humphreys. Review grain and subgrain characterisation by electron backscatter diffraction. Journal of Materials Science, 36:3833-3854, 2001.

[12] Z. Zhao, M. Ramesh, D. Raabe, A.M. Cuitino, and R. Radovitzky. Investigation of threedimensional aspects of grain-scale plastic surface deformation of an aluminum oligocrystal. International Journal of Plasticity, 24(12):2278 - 2297, 2008.

[13] A. Saai, H. Louche, L. Tabourot, and H.J. Chang. Experimental and numerical study of the thermo-mechanical behavior of al bi-crystal in tension using full field measurements and micromechanical modeling. Mechanics of Materials, 42(3):275 - 292, 2010.

[14] C. Badulescu, M. Grédiac, H. Haddadi, J.-D. Mathias, X. Balandraud, and H.-S. Tran. Applying the grid method and infrared thermography to investigate plastic deformation in aluminium multicrystal. Mechanics of Materials, 43(1):36 - 53, 2011.

[15] L. Bodelot, E. Charkaluk, L. Sabatier, and P. Dufrénoy. Experimental study of heterogeneities in strain and temperature fields at the microstructural level of polycrystalline metals through fully-coupled full-field measurements by digital image correlation and infrared thermography. Mechanics of Materials, 43(11):654 - 670, 2011.

[16] R. Seghir, J-F. Witz, L. Bodelot, E. Charkaluk, and P. Dufrénoy. An improved lagrangian thermography procedure for the quantification of the temperature fields within polycrystals. Quantitative InfraRed Thermography Journal, 10(1):74-95, 2013.

[17] L. Li, J.-M. Muracciole, L. Sabatier, L. Waltz, and B. Wattrisse. Analysis of the thermomechanical behavior of coarse-grained polycrystalline aluminum under tensile conditions. In PhotoMechanics 2013, Montpellier, France, 2013.

[18] S. Wen. Identification expérimentale de modèles de zones cohésives à partir de techniques d'imagerie thermomécanique. These, Université Montpellier II - Sciences et Techniques du 
Languedoc, December 2012.

[19] A. Chrysochoos, V. Huon, F. Jourdan, J.-M. Muracciole, R. Peyroux, and B. Wattrisse. Use of full-field digital image correlation and infrared thermography measurements for the thermomechanical analysis of material behaviour. Strain, 46(1):117-130, 2010.

[20] Li Li, Latoure Félix, Jean Michel Muracciole, Laurent Waltz, Laurent Sabatier, and Bertrand Wattrisse. Capturing polycrystal plasticity and intergranular cracks with a novel dic method. WCCM XI, Barcelona, Spain, 2014.

[21] V. Honorat, S. Moreau, J.-M. Muracciole, B. Wattrisse, and A. Chrysochoos. Calorimetric analysis of polymer behaviour using a pixel calibration of an irfpa camera. Quantitative InfraRed Thermography Journal, 2(2):153-171, 2005.

[22] A. Chrysochoos, O. Maisonneuve, G. Martin, H. Caumon, and J.C. Chezeaux. Plastic and dissipated work and stored energy. Nuclear Engineering and Design, 114:323-333, 1989.

[23] A. Chrysochoos and H. Louche. An infrared image processing to analyse the calorific effects accompanying strain localisation. International Journal of Engineering Science, 38(16):1759 $-1788,2000$.

[24] A. Blanche. Effets dissipatifs en fatigue à grand et très grand nombre de cycles. These, Université Montpellier II - Sciences et Techniques du Languedoc, December 2012.

[25] L. Meric, P. Poubanne, and G. Cailletaud. Single crystal modeling for structural calculations. i, model presentation. Journal of mechanical design (1990), 113(1):162-170, 1991. eng.

[26] F. Latourte, N. Rupin, and J.-M. Proix. Plasticité cristalline dans un acier bainitique revenu : simulations pour la validation de modèles à partir de mesures de champs. In 11e Colloque National en Calcul des Structures, France, May 2013. 